
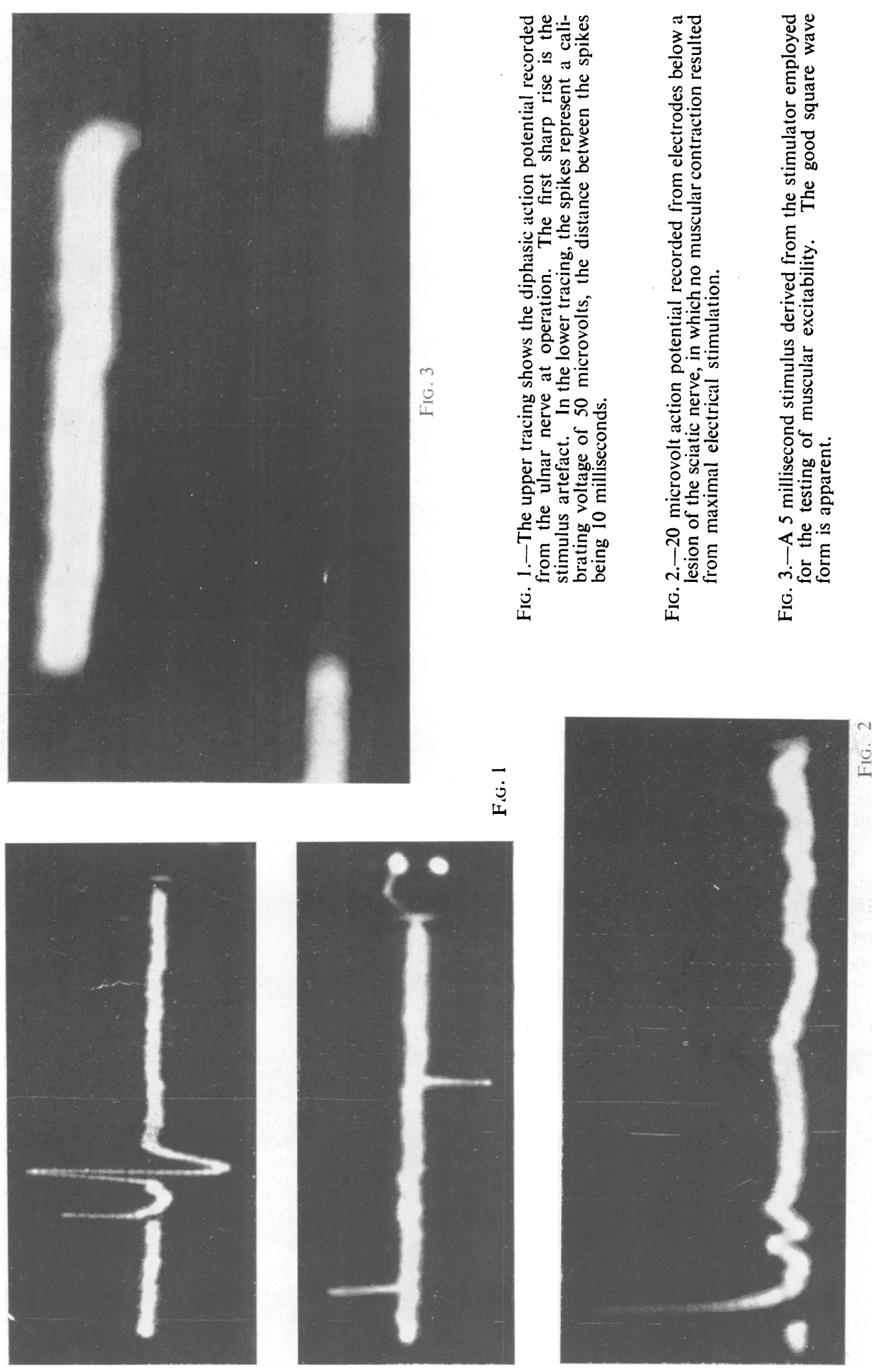


\title{
ELECTRICAL AIDS IN PROGNOSIS OF NERVE INJURIES
}

\author{
BY
}

\author{
H. W. NEWMAN and W. K. LIVINGSTON \\ From the Department of Medicine, Stanford University School of Medicine and the Department of Surgery, University \\ of Oregon Medical School
}

(ReCeIVed 24th August, 1947)

Peripheral nerve injuries resulting from wounding with high velocity missiles may vary from complete disruption of nerve trunks to a mild stretching of nerves at a distance from the pathway of the projectile. When such stretching is sufficiently severe as to interrupt the individual nerve fibres, the resulting paralysis may be most difficult to distinguish from that resulting from anatomic severance of the nerve trunk, yet the prognosis and treatment are quite different. In our experience - with 919 cases of nerve injury due to missile wounding there were four or five such " lesions in continuity" for every one with complete severance of the nerve. From our prolonged observation of patients with lesions in continuity, it was apparent that some of these lesions offered an insuperable obstacle to the progress of regenerating fibres, while many others spontaneously recovered a better degree of function than we could have hoped to confer by resection of the lesion and the most meticulous anastomosis.

Even when a lesion in continuity is exposed in the surgery it may be difficult to evaluate its chances for spontaneous regeneration. If electrical stimulation above the lesion results in contraction of muscles supplied by the nerve below the lesion, one may be confident that regeneration has taken place, but frequently the interval between wounding and surgical exposure of the lesion is too short to permit growing fibres to have reached the muscles which they supply.

\section{Nerve Action Potential Studies}

On the basis that regenerating nerve fibres should have established the property of conduction as they advanced toward the muscle, even before having achieved their goal, we endeavoured to pick up action potentials from the distal segment of the $\mathcal{O}^{\circ}$ injured nerve while stimulating electrically proximal to the lesion. Berry and others (1944) had studied action potentials in the regenerating sciatic nerve of the cat after section and suture. They found the $i$ threshold of the regenerating fibres to be muchio higher, and their rate of conduction slower, the in the normal fibres, these factors approachirat normal as the axonal calibre, studied histologicalle increased with time.

We derived the stimulating current from a Modelo Grass stimulator, passed through an electrostatica shielded transformer to minimize stimulus artefact, api delivered at the rate of 6 stimuli per second, diphastc. $V$. spikes of from 0.05 to 0.2 milliseconds being used. Both stimulating and recording electrodes were of 24-gauge? silver wire, each pair being embedded in a block of plastic $\supset$ material fashioned with a trough in which the nerve could rest and be insulated from the surrounding tissues. $\frac{\mathrm{O}}{8}$ The stimulating electrodes were placed above the lesion, $\varrho$ and the recording electrodes below. Action potentials $\overrightarrow{\vec{B}}$ were amplified, and visualized by a DuMont type 208-BЭ . cathode ray oscillograph. The sweep of the oscillograph was triggered by the same current which triggered the stimulator, permitting visualization of the stimulus. artefact as well as the action potential. Photography was accomplished by the use of a reflex camera with ao shutter speed of about $1 / 10$ second.

In all cases in which muscular contraction was obtainable on stimulation with the Hinsey-Geohagan? stimulator we were able to record action potentials from the nerve below the lesion. The type of record secured is illustrated in Fig. 1 (p. 99), which was taken at the time the ulnar nerve was transplanted at the elbow. The stimulating electrodes N were placed just above the scarred portion of the 
nerve, and the recording electrodes $11 \mathrm{~cm}$. below. The diphasic action potential, 150 microvolts from peak to peak, followed the stimulus artefact by approximately 3 milliseconds.

Fig. 2 (p. 99) shows the 20-microvolt action potential recorded peripheral to a lesion of the sciatic nerve in the mid-thigh. In this case no muscular movement could be secured on electrical stimulation, so that the action current must have been due either to regenerating fibres which had not yet reached the muscle, or conceivably to surviving sensory fibres. The nerve was subsequently sectioned and sutured, and a few apparently intact fibres were visible in the cut ends. Unfortunately we have no cases in which action potentials were secured without the presence of muscular contraction on electrical.stimulation, which have been followed to return of function without operative intervention. Thus we can only say of this method of investigation that it holds promise of clinical usefulness, but can present no concrete evidence of the extent to which it may be utilized at the present time.

\section{Neuromuscular Excitability Studies}

Another problem relating to regeneration on which the surgeon would like technical assistance is the question às to whether or not nerve fibres are growing across a suture line. After a nerve anastomosis has been performed it may be many months before growing fibres can reach the muscles and establish connexions adequate to make voluntary contractions possible. Any technical method which might shorten this interval of waiting for the demonstration of voluntary control should be of value to the surgeon. It $h$ has been well established that regenerating nerve fibres reach the muscle supplied a considerable period before overt motor power makes its appearance. It has been held that this time may be determined by the appearance of changes in the electrical excitability of the muscle which then take place. Should this be the case, valuable information regarding the prognosis might be secured at an appreciably earlier date than it could be ascertained by clinical examination alone. Many methods for determining this change in excitability have been advanced, each with its advocate.

Thus Moldaver (1942) is enthusiastic about chronaxie, while Bauwens (1944) believes chronaxie to be misleading, and prefers strength-duration curves. Ritchie (1944) and Marble and others (1942) use voltage-duration curves, and Pollock and others (1945) believe the galvanic tetanus ratio is valuable. In view of the wide divergence of opinion among different workers in the field as to the relative value of the various procedures, we felt it was impossible to decide which method would afford the' most reliable information unless several prognostic tests were carried out simultaneously on each patient. Those procedures which we employed were rheobase and chronaxie, strengthduration curve, galvanic tetanus ratio, and relationship between anodal and cathodal closing contraction threshold.

To this end an apparatus was constructed that was capable of producing the stimuli required for these tests. It has been described fully elsewhere (Newman, 1946), and consists essentially of a source of square waves of current, the duration and intensity of which are subject to control by the operator. Polarity is capable of reversal, and the current through the tissues is independent of skin resistance.

The course of 33 traumatic nerve lesions, mostly resulting from bullet and shrapnel wounds, was followed to recovery of definite motor function. Of these cases twenty-four were subjected to nerve suture, the remaining nine recovering spontaneously. Eighteen of the lesions were of the ulnar nerve, ten of the median, four of the radial, two of the peroneal, and one of the tibial. In general, the muscle chosen for electrical testing was one as remote as possible from the site of wounding ; in the case of the ulnar nerve the abductor of the little finger was used as a routine, and for the median the opponens. By picking out one particular muscle, and following its electrical excitability from the initial examination until motor function returned, it was felt that a better comparison between the various methods of testing could be made than by testing all of the paralyzed muscles. An attempt was made to repeat the tests at intervals of not greater than two weeks, but furloughs and intercurrent operative procedures sometimes prevented this.

The testing was conducted in a warm room, the temperature never falling below $70^{\circ} \mathrm{F}$. The indifferent electrode, a piece of cotton felt backed with lead sheet, about 10 by $10 \mathrm{~cm}$., was moistened with saline and strapped to the extremity to be tested, above the lesion if possible. The stigmatic electrode was chamois covered, of an area of about 1 sq.cm. It was kept moist with saline, and applied to the point on the muscle which showed the maximum response to stimulation. Thereafter, this same point was employed in subsequent testing.

The threshold was first determined for stimuli of 1,000 milliseconds' duration, the stigmatic electrode being the cathode. Then successively shorter stimuli were used, and each threshold intensity recorded, until no muscular contraction was obtained with full output of $20 \mathrm{ma}$. The resulting values, plotted as intensity of stimulus against duration of stimulus, constitute the strength-duration curve. When the duration is plotted 
on a logarithmic scale, curves are obtained such as are shown in the graph. Fig. 3 (p. 99) is an oscillographic record of the form of the stimulus obtained from the apparatus, which it will be noted is essentially square in conformation. Chronaxie was determined from the strength-duration curve by reading off the duration of stimulus at twice the rheobasic value by interpolation. The threshold intensity required to produce a single twitch at 1,500 milliseconds' duration of stimulus was next determined, and then the current was gradually increased until a contraction sustained throughout the entire time of passage of the current was obtained. This latter value divided by the former constitutes the galvanic tetanus ratio. In normal muscle this is about 4 , while in denervated muscle it approaches 1 . Polarity was then reversed, and the threshold for a single twitch with the anode as the stigmatic electrode recorded, to be compared with the threshold already obtained for cathodal closing contraction. The whole procedure took only a few minutes, and was well tolerated by the patients.

Analysis of the results obtained on repeated testing during the period of reinnervation demonstrated that all the tests employed showed a tendency in the majority of the cases to revert to the normal picture. Thus the extremely long values of chronaxie became shorter, and the reversed relationship between anodal and cathodal thresholds righted itself, while the rheobase tended to rise. However, in all the above-mentioned observations there was such a great variation in results from one examination to the next, with no abrupt change that could be depended upon as an indicator of reinnervation, that they were for all practical purposes useless in establishing prognosis.

Both the galvanic tetanus ratios and the strengthduration curves were, however, of real value in prognosis, as will be demonstrated by the following data. Of the cases followed to return of function, $\vec{\equiv}$ 54 per cent. showed a break, or discontinuity, in the strength-duration curve before motor power was re-established, the average time that the electrical change anticipated the clinical evidence of recovery being 97 days. Forty-eight per cent. of the cases showed a rise in the galvanic tetanus ratio to 3.0 है or higher, which value we believe to be the lowest that can be used safely as a criterion of recovery, an average of 109 days before return of motor power. In 30 per cent. of the cases both tests showed a change before motor recovery, yet on the other hand 27 per cent. had established motor

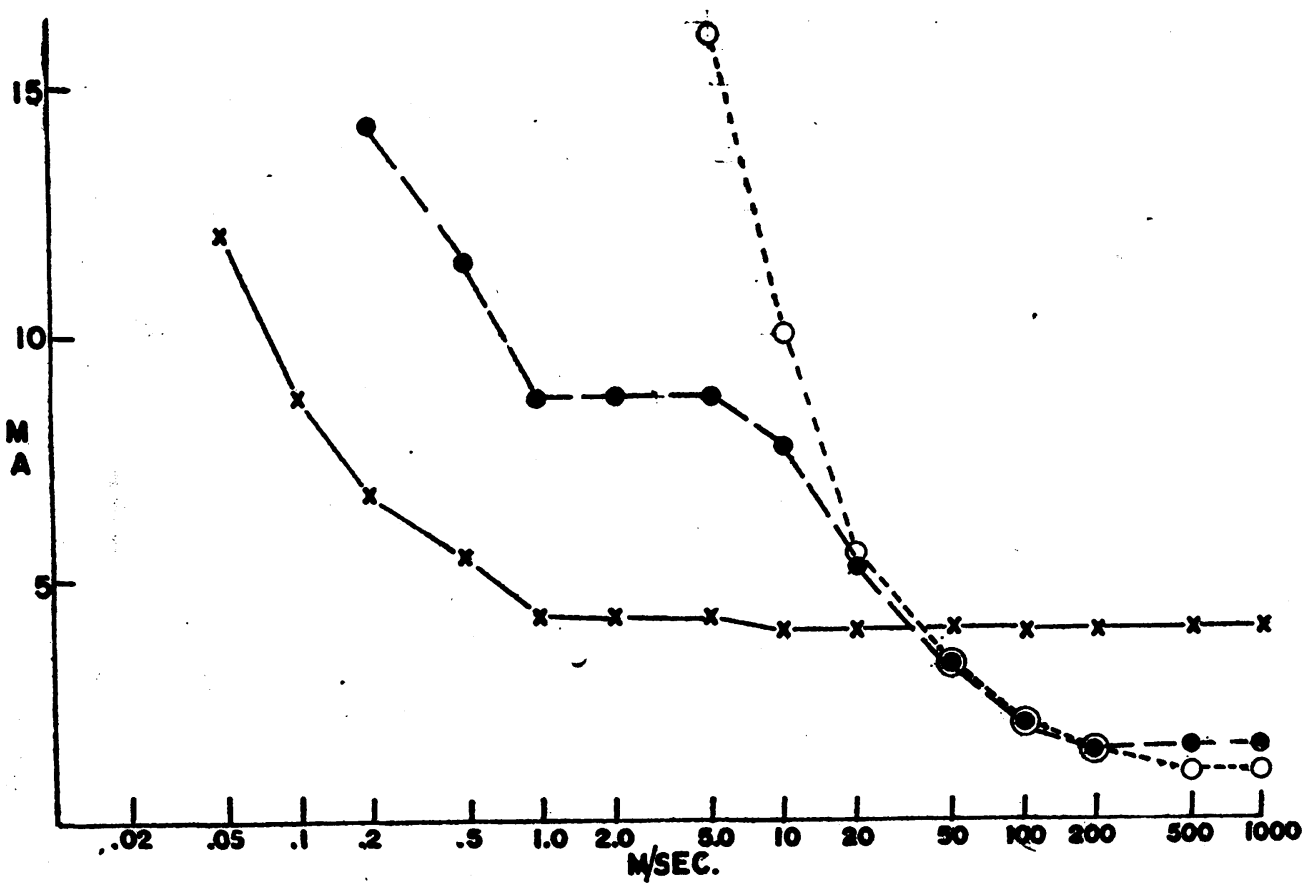

Strength-duration curves obtained from the abductor of the little finger. The open circles show the initial reaction of the muscle, the closed or solid circles that prevailing during recovery, with a break in the mid-portion of the curve. The crosses show the curve obtained from the normal muscle of the opposite side. 
function before any reliable evidence of electrical change was apparent. Thus it can be seen that with either one or the other of the two tests reliable signs of recovery were obtainable in about half the cases, while if both were employed this was raised to 73 per cent. Therefore, although in an appreciable percentage of the cases the tests failed completely to reveal the progress of reinnervation, in by far the majority they yielded information of considerable prognostic value.

Thus far only those cases which proceeded to motor recovery under observation have been considered. It is equally important to consider those in which we have electrical data but in which a lack of return of motor function might indicate failure of regeneration, in order to ascertain if the electrical tests may yield false evidence of recovery. In this connexion thirteen lesions, of which ten were ulnar, two median, and one radial, were followed for approximately one year without appearance of motor recovery. In four of these there was no electrical change to indicate regeneration, so these may be dismissed from further consideration. Of the remaining nine cases, two showed favourable electrical changes in both tests, five a rise in the galvanic tetanus ratio, and two a break in the strength-duration curve. In four of these cases the period of observation had been twice as long at least as the interval from time of suture to appearance of electrical changes, so that ultimate functional recovery seemed unlikely. It seems quite possible, however, that, although sufficient fibres had not regenerated to produce an active muscular contraction, a sufficient number had grown down to produce the electrical changes noted. In the remaining five cases the period of observation was not long enough to be sure that motor power might not yet appear. In only one case did electrical changes appear before theoretical considerations would permit of fibres reaching the muscle; this case must surely be considered a false positive sign from the electrical standpoint. However, it can be seen thàt in a not inconsiderable number of cases positive electrical signs of recovery were observed without actual return of useful motor function.
This seriously detracts from the value of the tests in prognosis, where such evidence must be weighed in determining whether or not operative intervention is indicated, since such false positive findings would then result in undue postponement of operation. When it is considered that even the degree of reliability of results above detailed was secured only by dint of frequently repeated examinations, it is at once apparent that the results of isolated single examinations must be much less valuable, and should not be given undue weight.

\section{Summary and Conclusions}

An attempt was made to record, by means of amplifiers and a cathode ray oscillograph, nerve action potentials from injured nerves to determine the presence or absence of regeneration. Although the method showed promise of usefulness, sufficient data are not yet at hand to permit of a fair appraisal.

Determination of the galvanic tetanus ratio, and of the form of the strength-duration curve, is of practical value in establishing the presence of regeneration an appreciable time before clinical examination would be adequate for the purpose. However, neither are these signs always present, nor can they invariably be relied upon to establish the return of useful motor function. Since it is of the utmost importance in the proper handling of peripheral nerve injuries to determine at as early a date as possible whether or not regeneration is taking place, the electrical methods which we have described, as well as electromyograms and any other procedure which might reasonably shed light on this problem, are amply justified.

\section{REFERENCES}

Bauwens, P. (1944). Arch. Phys. Therap., 25, 288.

Berry, C. M., Grundfest, H., and Hinsey, J. C. (1944). J. Neurophysiol., 7, 103.

Marble, H. C., Hamlin, E., and Watkins, A. L. (1942). Amer. J. Surg., 55, 274.

Moldaver, J. (1942). J. Dis. Nerv. Syst., 3, 20.

Newman, H. W. (1946). Proc. Soc. exp. Biol., N.Y. 63, 201.

Pollock, L. J., Golseth, J. G., and Arieff, A. J. (1945). Surg. Gynec. Obstet. 81, 660 .

Ritchie, A. E. (1944), Brain, 67, 314.

\section{For half-tone illustrations to this article see page 99}

